\title{
VIBURNUM OPULUS L. VE VIBURNUM LANTANA L.'DA AMENTOFLAVON HPLC ANALIZI
}

\author{
HPLC ANALYSIS OF AMENTOFLAVONE IN VIBURNUM OPULUS L. AND \\ VIBURNUM LANTANA L.
}

\section{Levent Altun, Betül Sever Yilmaz, Gülçin Saltan ÇítoĞLU}

Ankara Üniversitesi Eczacılık Fakültesi Farmakognozi Anabilim Dalı, 06100, TandoğanANKARA

\begin{abstract}
ÖZET
Bu çalışmada Viburnum opulus ve V. lantana yaprak, dal ve meyvalarında amentoflavon'un kantitatif analizi için basit ve duyarlı bir yöntem kullanılmıştır. Amentoflavon'un miktar tayini Supelcosil LC 18 (250x4.6 mm, $5 \mu \mathrm{m})$ kolonunda asetonitril: su : fosforik asit (52: 47: 1) (h/h/h) solvan sistemi kullanılarak yapılmıştır. Amentoflavon miktarl; V. lantana yapraklarında \% 0.1104, V. lantana dallarında \% 0.0061 olarak bulunmuştur. V. opulus yapraklart için bu değer \% 0.0066 olarak belirlenmiştir. V. opulus'un dal ve meyvalarında, $V$. lantana'nın ise meyvalarında amentoflavona rastlanmamıştır.

Anahtar Kelimeler : Amentoflavon, V. opulus, V. lantana, HPLC, yapraklar, dallar, meyvalar

\section{ABSTRACT}

In this study a simple and sensitive HPLC method for separation and quantitative determination of amentoflavone in the leaves, the branches and the fruits of Viburnum opulus L. and Viburnum lantana L. has

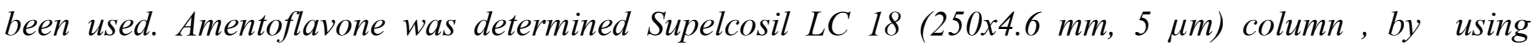
acetonitrile: water : phosphoric acid (52: 47: 1) (v/v/v) as a mobile phase. The amentoflavone content of $V$. lantana was found to be $0.1104 \%$ for leaves, $0.0061 \%$ for branches. For V. opulus leaves, this value was determined as $0.0066 \%$. Neither branches and fruits of Viburnum opulus nor fruits Viburnum lantana posses amentoflavon.
\end{abstract}

Key Words: Amentoflavone, V. opulus, V.lantana, HPLC, leaves, branches, fruits 


\section{GíRiş}

Caprifoliaceae familyasına ait Viburnum cinsi Güney Amerika'dan Güney Doğu Asya'ya kadar geniş bir dağılım gösteren ve çoğu endemik olan 230'dan fazla tür içermektedir (1).

Bitki Türkiye Florasında dört türle temsil edilmektedir; Viburnum opulus L., V. orientale Pallas, V. lantana L. ve $V$. tinus L. (2,3).

$V$. opulus idrar arttırıcı, müshil ve yatıştırıcı etkilere sahiptir. Safra ve karaciğer hastalıklarına karşı Orta Anadolu bölgesinde kırmızı renkli meyvaların (gilaburu meyvası) usaresi kullanılmaktadır. Meyvalar yemiş olarak da yenilmektedir. V. lantana'nın taze dal kabukları ise haricen kızartıcı ve ağrı kesici olarak kullanılmaktadır (4). $V$. dilatatum bitkisinin oksidatif hasarlara karşı koruyucu etkisi strese maruz kalmış sıçanlar ve streptozotosin ile uyarılmış diyabetik sıçanlarda gösterilmiştir $(5,6)$. Ayrıca $V$. dilatatum bitkisinin plazma, karaciğer ve midedeki antioksidan enzimler üzerine etkisi incelendiğinde bu bitkinin kullanılması süperoksit dismutaz, katalaz ve glutatyon peroksidaz gibi antioksidan enzimlerin tüketiminin azalmasına katkıda bulunabilmektedir (7). V. erubescens Wall. bitkisinin alkollü ekstresinin düşük antiviral etkiye sahip olduğu gösterilmiştir (8). V. luzonicum'dan izole edilen bazı iridoit aldehitlerin He La S3 kanser hücrelerine karşı orta derecede inhibitör etki gösterdiği bulunmuştur (9). Viburnum cinsine ait türlerin triterpen (10-11), diterpen (12,13), seskiterpen (14) ve iridoit (15-19) yapısında bileşikler taşıdığı bilinmektedir. Ayrıca Viburnum grandifolium'dan luteolin 3'-O- $\beta$-ksilozil glukozit ve apigenin 7- ksilozil glukozit izole edilmiştir (20). Farklı Viburnum türlerinde Yapılan kemotaksonomik çalışmalar sonucunda biflavonoit yapısında olan amentoflavon tesbit edilmiştir $(1,21)$.

Bir apigenol dimeri olan amentoflavonun antifungal, antienflamatuvar ve antioksidan etkilerinin olduğu bilinmektedir (22).

$\mathrm{Bu}$ çalışmada Viburnum opulus ve $V$. lantana yaprak, dal ve meyvalarının metanollü ekstrelerinde biflavonoit yapısında olan amentoflavonun ayırımı ve miktar tayini için basit ve duyarlı bir yöntem kullanılmıştır (23). 


\section{MATERYAL VE METOD}

\section{Bitki Materyali}

Viburnum opulus Kayseri'den (AEF 23696), Viburnum lantana ise Ankara'dan (AEF 23543) toplanmıştır.

\section{Ekstrenin Hazırlanışı}

$V$. opulus ve $V$. lantana bitkilerinin yaprak, dal ve meyvaları kurutulup toz edildikten sonra 5'er gram tartılmıştır. Oda sıcaklığında 1'er saat süre ile $100 \mathrm{ml}$ metanolle ultrasonik banyoda ekstre edilmiştir. Süzüldükten sonra tüm ekstrelerin hacmi balon jojede 100 ml'ye tamamlanmış ve iyice çalkalanmıştır. Bu ekstreler $0.45 \mu$ m'lik filtreden geçirilip $20 \mu$ miktarda, cihaza entegre edilmiş otomatik şırınga ile hassas olarak HPLC kolonuna enjekte edilmiştir.

\section{Cihaz}

Yüksek Basınçlı Sıvı Kromatograf olan HP-1100 (Agilent Technologies, Inc., California, USA) cihazı kullanılmıştır. Pik alanları Agilent software bilgisayar programı tarafından otomatik olarak hesaplanmıştır.

\section{Kromatografik Şartlar}

Mobil Faz: Asetonitril: Su : Fosforik asit (52: 47: 1) (h/h/h) (23).

HPLC saflığında Asetonitril (Merck-100030), Formik asit (Merck-100264) ve kromatografik saflıkta bidistile su kullanılmıştır. Solvanlar $0.45 \mu \mathrm{m}$ filtreden (Milipore, Milfod, USA) geçirilip ultrasonik su banyosunda degaze edilmiştir.

Akış Hızı: 1ml/dak.

Dedektör: 330 nm (Diod-Array Dedektör)

Kolon: Supelcosil LC $18(250 \times 4.6 \mathrm{~mm}, 5 \mu \mathrm{m})$

\section{Standart Çözeltinin Hazırlanması}

Amentoflavon (40584) standardı Fluka firmasından temin edilmiştir.

Amentoflavon (5 mg) standardı metanolle çözülüp balon jojede 10 ml'ye tamamlanmıştır. Böylece $500 \mu \mathrm{g} / \mathrm{ml}$ 'lik standart çalışma çözeltisi hazırlanmıştır. Analizler eksternal standart yöntemi kullanılarak yapılmıştır. Bunun için de; standart çalışma çözeltisinden $1 \mathrm{ml}$ alınıp 10 ml'ye $(50 \mu \mathrm{g} / \mathrm{ml}), 2 \mathrm{ml}$ alını $10 \mathrm{ml}$ 'ye $(100 \mu \mathrm{g} / \mathrm{ml}), 3 \mathrm{ml}$ alınıp $10 \mathrm{ml}$ 'ye $(150 \mu \mathrm{g} / \mathrm{ml}), 4 \mathrm{ml}$ alınıp $10 \mathrm{ml}$ 'ye $(200 \mu \mathrm{g} / \mathrm{ml})$ tamamlanmıştır. Daha sonra $50 \mu \mathrm{g} / \mathrm{ml}$ konsantrasyondaki çözelti yarı 
yarıya $(25 \mu \mathrm{g} / \mathrm{ml})$ ve $150 \mu \mathrm{g} / \mathrm{ml}$ konsantrasyondaki çözelti de $1 / 100$ oranında $(1.5 \mu \mathrm{g} / \mathrm{ml})$ seyreltilerek farklı toplam 6 dilusyon hazırlanmıştır (1.5- $200 \mu \mathrm{g} / \mathrm{ml})$.

\section{Kalibrasyon Eğrisi}

Metanol içinde hazırlanan amentoflavon (1.5- $200 \mu \mathrm{g} / \mathrm{ml})$ standardına ait kalibrasyon grafiği elde edilmiştir (Grafik 1).

Amentoflavon'un farkl1 6 konsantrasyonundan 3'er kez 20'şer $\mu 1$ enjekte edilmesi ile pik alanları saptanmış ve buradan $\mathrm{y}=\mathrm{mx}+\mathrm{n}$ doğru denklemi bulunmuştur.

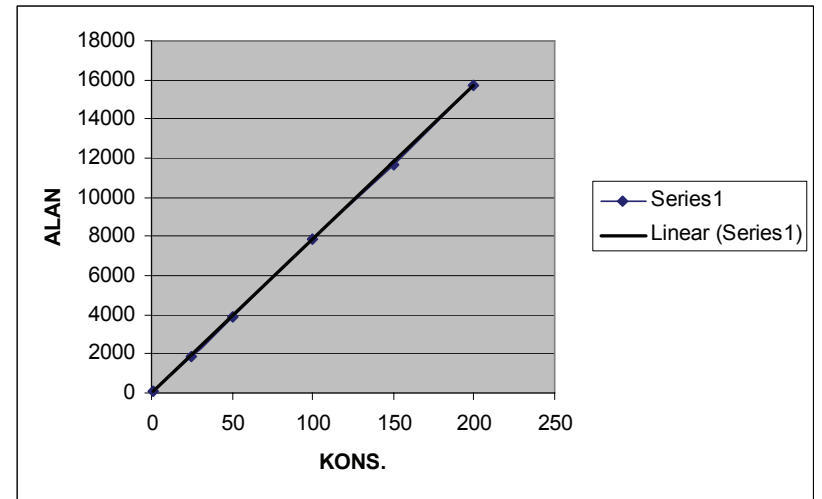

Grafik 1: Amentoflavon'un Kalibrasyon Eğrisi<smiles></smiles>

Amentoflavon

\section{BULGULAR}

Krauze-Baranowska ve arkadaşları (23) tarafından geliştirilen yönteme göre; Asetonitril: Su: Fosforik asit (52: 47:1) $(\mathrm{h} / \mathrm{h} / \mathrm{h})$ solvan sistemi kullanılarak $1 \mathrm{ml} /$ dak. akış hızında ters faz kolonunda, UV $330 \mathrm{~nm}$ 'de amentoflavon standard1 maksimum bir absorbans göstermiş olup retansiyon zamanı 4.8 dakika olarak tesbit edilmiştir (Kromatogram 1). 


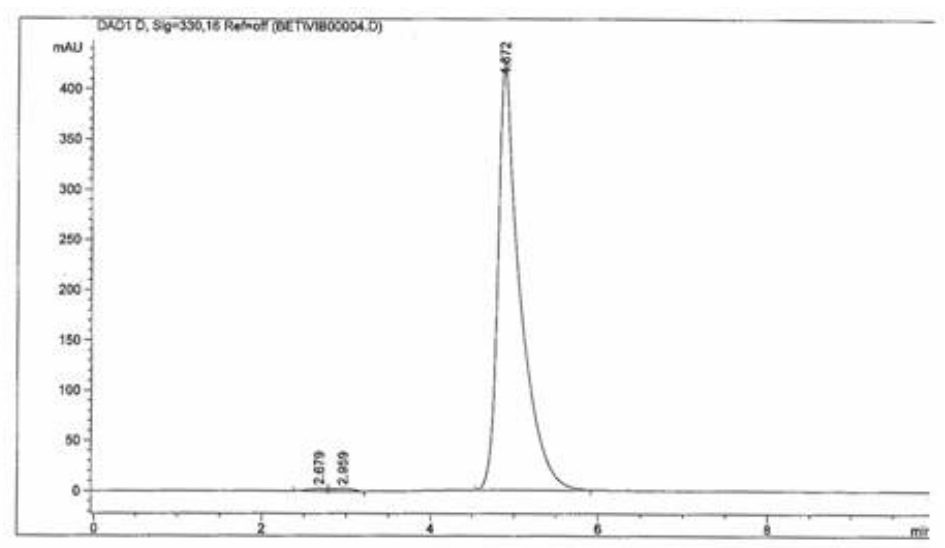

Kromatogram 1: Standart Amentoflavon'un HPLC Kromatogramı

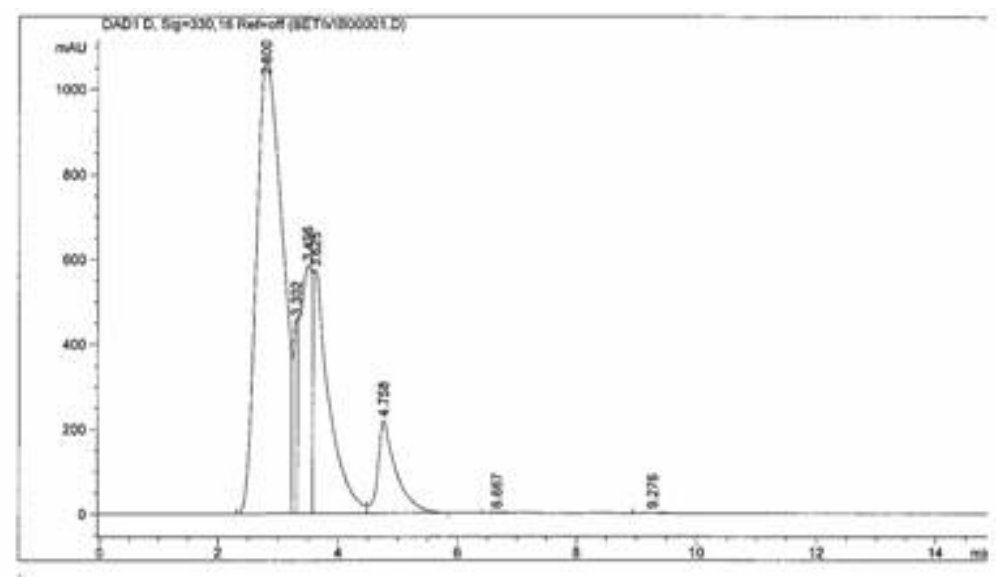

Kromatogram 2: Viburnum lantana yaprak ekstresinin HPLC Kromatogramı (Amentoflavon $\mathrm{Rt}=4.758$ )

\section{Doğrusallık}

Tablo 1'de amentoflavon standardına ait korelasyon değeri (r2), eğim ve kesişimin bağıl standart sapmaları verilmiştir. Amentoflavonun 1.5-200 $\mu \mathrm{g} / \mathrm{ml}(\mathrm{r} 2=0.9998)$ konsantrasyonlarda elde edilen pik alanları ile mükemmel bir doğrusallık sağlanmıştır.

Teşhis Limiti ve Miktar Tayini Limitlerinin Saptanması

Teşhis edilebilecek en düşük miktar (TL) gürültü sinyalinin 3 katı, miktar tayini yapılabilecek en düşük miktar (MTL) gürültü sinyalinin 9 katı olarak bulunmuştur. Buna göre de amentoflavon için 6 enjeksiyon yapılmış olup teşhis edilebilecek en düşük miktar $0.5 \mu \mathrm{g} / \mathrm{ml}$; miktar tayini yapılacak en düşük miktar da $1.5 \mu \mathrm{g} / \mathrm{ml}$ olarak hesaplanmıştır (Tablo 1). 
Tablo 1: Sonuçların Doğrusallığı, Teşhis Limiti ve Miktar Tayin Limiti

\begin{tabular}{|l|c|c|c|c|c|c|c|}
\hline Bileşik & $\lambda$ & Doğru Denklemi & $\mathrm{r}^{2}$ & $\begin{array}{c}\text { Eğim } \\
(\% \mathrm{BSS})\end{array}$ & $\begin{array}{c}\text { Kesişim } \\
(\% \mathrm{BSS})\end{array}$ & $\begin{array}{c}\mathrm{MTL} \\
(\mu \mathrm{g} / \mathrm{ml})\end{array}$ & $\begin{array}{c}\mathrm{TL} \\
(\mu \mathrm{g} / \mathrm{ml})\end{array}$ \\
\hline Amentoflavon & 330 & $\mathrm{Y}=78.665 \mathrm{X}+58.214$ & 0.9998 & 0.071 & 2.545 & 1.5 & 0.5 \\
\hline
\end{tabular}

$\mathbf{X}=$ Konsantrasyon $(\mu \mathrm{g} / \mathrm{ml}) ; \mathbf{Y}=$ Alan; $\lambda=$ Dalga Boyu;

$\mathbf{r}^{2}=$ Korelasyon değeri; $\%$ BSS $=\%$ Bağ 1 L Standart Sapma;

MTL= Miktar Tayin Limiti

$\mathbf{T L}=$ Teşhis Limiti

\%BSS $=($ Standart Sapma/ Ortalama $) \times 100$

\section{Doğruluk}

Metodun doğruluğu amentoflavon standardının miktar tayini yapılabilecek en düşük konsantrasyonundan $9 \mathrm{kez}$ enjekte edilerek kanıtlanmıştır. Ayrıca amentoflavonun miktar tayini yapılabilecek en düşük miktardaki bağıl standart sapması da \% 0.931 olarak hesaplanarak kullanılan bu metodun doğruluğu kesinleştirilmiştir (Tablo 2).

Tablo 2: Metodun Miktar Tayin Limitindeki Doğruluk Değeri $(n=9)$

\begin{tabular}{|c|c|c|c|}
\hline Bileşik & $\lambda$ & $\begin{array}{c}\text { Pik Alanı } \\
(\mathrm{n}=9, \text { ortalama })\end{array}$ & $\% \mathrm{BSS}$ \\
\hline Amentoflavon & 330 & 107.43 & 0.931 \\
\hline
\end{tabular}

\section{V. opulus ve V. lantana Ekstrelerinde Amentoflavon Bileşiğinin Analizi}

Kayseri ve Ankara'dan toplanan V. opulus ve V. lantana bitkilerinin yaprak, dal ve meyvalarından hazırlanan ekstrelerde ters faz HPLC ile amentoflavon bileşiğinin teşhisi yapılmıştır. Ekstrelere amentoflavon standardı tek bir konsantrasyonda $(50 \mu \mathrm{g} / \mathrm{ml})$ ayrı ayrı ilave edilip ayrı ayrı 3'er defa enjeksiyon yapılarak adı geçen bileşiğe ait retansiyon zamanlarında piklerde büyüme olduğu tespit edilmiştir. Böylece ekstrelerde amentoflavon bileşiğinin varlığ kesinleştirilmiştir. Sonuçlar istatistiksel olarakda değerlendirilerek bu standarda ait doğru denkleminden hareketle ekstrelerdeki \% miktarı hesaplanmıştır (Tablo 3). 
Tablo 3: Viburnum Türlerinde Bulunan \% Amentoflavon Miktarları

\begin{tabular}{|c|c|c|}
\hline Türler & $\begin{array}{c}\text { Alan } \\
(\mathrm{n}=3, \text { ortalama })\end{array}$ & $\begin{array}{c}\text { \% Amentoflavon } \\
\text { Ortalama } \pm \mathrm{SS}\end{array}$ \\
\hline V. opulus & 199.96 & $0.0066 \pm 0.0002$ \\
yaprak & $(3.777)$ & $(3.517)$ \\
\hline V. lantana & 4283.94 & $0.1104 \pm 0.0006$ \\
yaprak & $(0.529)$ & $(0.543)$ \\
\hline V. lantana dal & 183.56 & $0.0061 \pm 0.0005$ \\
& $(2.049)$ & $(0.941)$ \\
\hline
\end{tabular}

*\%BSS = Parentez içinde \% Bağıl Standart Sapma değerleri verilmiştir.

$\mathbf{S S}=$ Standart Sapma

\section{TARTIŞMA}

Yapılan analizler sonucunda $V$. opulus yapraklarında \% 0.0066, $V$. lantana yapraklarında $\% 0.1104$ (Kromatogram 2) ve $V$. lantana dallarında \% 0.0061 oranında amentoflavon bulunmasına rağmen $V$. opulus dal, meyva ve $V$. lantana meyvalarında amentoflavona rastlanmamıştır. Lobstein ve arkadaşları tarafından yapılan bir çalışmaya göre amentoflavon miktarları; V. lantana yapraklarında $\% 0.578$, dallarında 0.026 , V. opulus yapraklarında $\% 0.042$, dallarında \% 0.010 olarak bulunmuştur (1).

Bizim çalışmamızda elde ettiğimiz sonuçların Lobstein ve arkadaşlarının elde ettiği sonuçlara göre daha az olmasının nedeni iklim koşulları, coğrafik farklılıklar gibi bir çok faktöre bağlanabilir.

Woo ve arkadaşlarının (22) yaptıkları çalışmaya göre biflavonoit yapısında olan amentoflavonun antifungal, antienflamatuvar ve antioksidan etkilerinin olduğu tesbit edilmiştir. Ancak çalıştı̆̆ımız Viburnum türlerinde amentoflavon miktarının daha önce çalışılan türlere (1) göre düşük çıkması ya da bazı kısımlarında hiç bulunmaması amentoflavona bağlı olarak görülen etkiler bakımından bu türlerin değerlendirilmesinin doğru olamayacağı kanısına varmamıza neden olmuştur.

Yine bu verilere göre çalıştığımız $V$. opulus ve $V$. lantana yaprak, dal ve meyvalarının amentoflavon kaynağı olarak değerlendirilmesinin ekonomik olamayacağı sonucuna varılmıştır. 


\section{KAYNAKLAR}

1. Lobstein, A., Haan-Archipoff G., Englert, J., Kuhry, J.G., Anton, R. "Chemotaxonomical investigation in the genus Viburnum" Phytochemistry, 50, 1175-1180 (1999).

2. Davis, P.H., Flora of Turkey and the East Aegean Islands, Edinburgh University Press, Edinburgh, Vol. 4, s.543 (1972).

3. Davis, P.H., Mill, R.R., Tan, K., Flora of Turkey and the East Aegean Islands, , Edinburgh University Press, Edinburgh, Vol. 10, s.154 (Supplement) (1988).

4. Baytop, T., Türkiye'de Bitkiler ile Tedavi (Geçmişte ve Bugün), 2. Baskı, Nobel Tip Kitabevleri, İstanbul, s. 210 (1999).

5. Gruenwald, J., Brendler, T., Jaenicke, C., PDR for herbal medicines, Medical EconomicsCompany, Montvale, New Jersey, s. 96-97 (2000).

6. Iwai, K., Onodera, A., Matsue, H. "Antioxidant acitivity and inhibitory effect of Gamazumi (Viburnum dilatatum THUNB.) on oxidative damage induced by water immersion restraint stress in rats" Int. J. Food. Sci. Nutr., 52(5), 443-451 (2001).

7. Iwai, K., Kim, M.Y., Onodera, A., Matsue,H. "Physiological effects and active ingredients of

Viburnum dilatatum Thunb fruits on oxidative stress" The proceedings of the 3rd International Conference on Food Factors (IcoFF 03), 21(1-4), 273-275 (2004).

8. Kim, M.Y., Iwai, K., Matsue, H. "Phenolic compositions of Viburnum dilatatum Thunb. Fruits and their antiradical properties" J. Food Compos. Anal., 18, 789-802 (2005).

9. Fukuyama, Y., Minoshima, Y., Kishimoto, Y., Chen,I., Takahashi, H., Esumi, T. "Cytotoxic iridoid aldehydes from Taiwanese Viburnum luzonicum" Chem. Pharm. Bull., 53(1), 125127 (2005).

10. Kagawa, M., Minami, H., Nakahara, M., Takahashi, H., Takaoka, S., Fukuyama, Y. “ Oleanane -type triterpenes from Viburnum awabuki" Phytochemistry, 47(6), 1101-1105 (1998).

11. Fukuyama, Y., Minami, H., Fujii, H., Tajima, M. " Triterpenoids from Viburnum suspensum" Phytochemistry, 60(8), 765-768 (2002).

12. Fukuyama, Y., Minami, H., Matsuo, A., Kitamura, K., Akızuki, M., Kubo, M., Kodama,M. "Seven- Membered vibsane- type diterpenes with a 5,10-cis relationship from Viburnum awabuki" Chem. Pharm. Bull., 50(3), 368-371 (2002). 
13. Fukuyama, Y., Kubo, M., Minami, H., Matsou, A., Fujii, T., Morisaki, M., Harada, K. "Rearranged vibsane-type diterpenes from Viburnum awabuki and phytochemical reaction of Vibsanin B"Chem.Pharm. Bull., 53(1), 72-80 (2005).

14. Fukuyama, Y., Minami, H., Ichikawa, R., Takeuchi, K., Kodama,M. "Hydroperoxylated guaiane-type sesquiterpenes from Viburnum awabuki" Phytochemistry, 42(3), 741-746 (1996).

15. Iwagawa,T., Yaguchi, S., Hase, T. "Iridoid Glycosides from Viburnum suspensum" Phytochemistry, 29(1), 310-312 (1990).

16. Iwagawa, T., Yaguchi, S., Hase, T. "Iridoid Glucosides From Viburnum suspensum" Phytochemistry, 35(5), 1369-1370 (1994).

17. Çalış, İ., Yürüker, A., Rüegger, H., Wright, A.D., Sticher, O. "Lantanoside, a monocyclic $\mathrm{C}_{10}$ iridoid glucoside from Viburnum lantana" Phytochemistry, 38(1), 163-165 (1995).

18. Tomassini, L., Foddai, S., Nicoletti, M., Cometa, M.F., Palazzino, G., Galeffi, C. “ Iridoid Glycosides from Viburnum ayavacense" Phytochemistry, 46(5), 901-905 (1997).

19. Tomassini, L., Gao, J., Serafini, M., Nicoletti, M. " Iridoid Glucosides from Viburnum sargenti" Natural Product Research, 19(7), 667-671 (2005).

20. Parveen, M., Khan, M.S., Ilyas, S., Ilyas, M. "Luteolin 3'-xylosyl $(1 \rightarrow 2)$ glucoside from Viburnum grandifolium" Phytochemistry, 49(8), 2535-2538 (1998).

21. Lobstein, A., Weniger, B., Malécot, V., Um, B.H., Alzate, F., Anton, R. "Polyphenolic content of two Colombian Viburnum species (Caprifoliaceae)" Biochem. Syst. Ecol., 31(1), 95-97 (2003).

22. Woo, E.R., Lee, J.Y., Cho, I.J., Kim, S.G., Kang,K.W. " Amentoflavone inhibits the induction of nitric oxide synthase by inhibiting NF-KB activation in macrophages" Pharmacological Research, 51, 539-546 (2005).

23.Krauze-Baranowska, M., Bączek, T., Glod, D., Kaliszan, R.,Wollenweber, E. "HPLC Separation of O- Acylated flavonoids and biflavones from some species of Gymnospermae" Chromatographia 60, 9-15 (2004). 
